\title{
Departamento de Ciências Médicas
}

\author{
Fernando Helio Alencar $\left({ }^{*}\right)$ \\ Roger Shrimpton ( $\left.{ }^{(}\right)$
}

Resumo

O Departamento Médico do INPA nasceu com o Instituto, em 27 de julho de 1954. Desde aquela data tem se dirigido a estudo dos problemas de saúde do homem da Amazônia. O histórico do Departamento é relatado, destacando três fases de atuação nas épocas dos Diretores: Djalma Batista, Paulo de Almeida Machado e Warwick Estevan Kerr. Os trabalhos são revisados em três áreas: estudos sobre os agentes das doenças da Amazônia; estudos sobre os vetores dos parasitas (sendo destacados os trabalhos de Nelson Cerqueira, sobre a transmissão da Mansonella ozzardi); e estudos relacionados ao homem. Conclui com a contribuição do Departamento ao desenvolvimento da Medicina Tropical e Saúde da Amazônia.

\section{INTRODUÇÃO}

O Instituto foi criado no dia 27 de julho de 1954, sob a direção do Professor Olympio da Fonseca. Justamente nesta data, o Departamento Médico do INPA dava início aos seus trabalhos, tendo como objetivo principal o estudo da saúde do homem amazônico. Para alcançar este objetivo, foi o Departamento Médico estruturado inicialmente com uma grande área de atuação, tendo as seguintes linhas de pesquisas: Microbiologia Médica, Imunologia, Parasitologia Geral e Médica, Fisiologia e Farmacologia, Higiene e Epidemiologia, Clínica e Patologia (Reis, 1958).

Os passos iniciais deste Departamento, foram inseguros e pontilhados de receios e muitas dúvidas. Obviamente, os seus primeiros trabalhos científicos são frutos de uma fase de ensaios e adaptações, entretanto, documentam um esforço persistente e honesto, de uma equipe de pessoas abnegadas, cujo objetivo único era o conhecimento da região Amazônica, e as condições de vida de sua coletividade.
Ao longo destes anos o Departamento sofreu várias modificações de nome e de estrutura. Inicialmente com o nome de Divisão de Biologia, sob a direção do Dr. Djalma Batista (entre 1957-1959) e depois com o Dr. Mário Moraes, quando o Dr. Djalma Batista era Diretor do instituto (1959-1968). Foi nesta época que se concretizaram as expedições à Codajás, para desenvolver estudos integrados dos problemas de saúde, naquele local (Batista; Oliveira \& Rabelo, 1960a). Participaram daquelas expedições, ilustres pesquisadores, tais como; Luís Montenegrc, Wallace Oliveira Ramos, Samuel Aguiar, Manoel Bastos Lira, Girginia Dupré Rabelo, Mário A. P. Moraes e Nelson L. Cerqueira. O alto grau de entendimento dos problemas da Amazônia apresentado pelo Dr. Djalma Batista, é mostrado no livro entitulado "O Complexo da Amazônia" (Batista, 1979).

O Diretor Dr. Paulo de Almeida Machado (1969-1972) deu ênfase para que o Departamento pesquisasse a Micologia Médica e a Hanseníase na Amazônia (Machado, 1971). Na sua gestão foi dado um dos passos mais importantes do Departamento, a construção de um pavilhão, para alojar a "Divisão de Patologia Tropical", dando assim condições para aumentar as atividades (o que vem acontecendo nas gestões sucessivas).

No período diretorial do Dr. Warwick E. Kerr (1975-1979), tendo o Dr. Jorge Ramón Arias como Chefe do Departamento de Ciências Médicas, a estrutura ativa baseava-se em; Parasitologia I e II (pesquisando a Leishmaniose: Malária e Filarioses), Micologia Médica, Mioléstias Gastro-Intestinais e Nutrição.

Diante da inexistência de um hospital no Instituto que servisse de suporte para o meIhor conhecimento clínico das várias patoiogias da região, as pesquisas do Departamento foram dirigidas às comunidades e aos campos da

(*) - Instituto Nacional de Pesquisas da Amazônia, Manaus. 
Amazônia, para entender quais dos fatores ambientais teriam importância na saúde do homem amazônico.

Para melhor avaliar a contribuição das pesquisás do Departamento ao desenvolvimento científico da Amazônia, decidimos analisar a literatura na base de três divisões, sendo; Estudos sobre os agentes das doenças; estudos sobre os vetores dos agentes; e estudos sobre o hospedeiro e o homem. Na bibliografia citada nem todos os trabalhos publicados pelo Departamento são incluídos. Acreditamos que existam trabalhos publicados em revistas estrangeiras, por pesquisadores do INPA após voltarem ao seu país de origem, dos quais ainda não tomamos conhecimento. Somente foram considerados os trabalhos já publicados ou aceitos para publicação.

\section{ESTUDOS SOBRE OS AGENTES DAS DOENÇAS DA AMAZONIA}

Dos parasitas de importância médica destacam-se os plasmódios, agentes etiológicos da malária. Deane e colaboradores pesquisaram a malária nos macacos da região (Deane et al., 1966). Num estudo colaborativo entre o INPA e o Hospital de Moléstias Tropicais de Manaus, Ferraroni \& Dourado (1977) pesquisaram o tratamento da malária por Plasmodium falciparum com minociclina endovenosa, mostrandio sua eficiência.

Fonseca et al. (1973) fizeram inquéritos imunoalérgico na Amazônia, achando entre 1213 reações intradérmicas, $32.97 \%$ positiva para Leishmania. Arias e colaboradores mostraram a ineficácia de uma vacina anti-Chagas contra duas cepas de Leishmania em Hamster (Arias et al., 1976).

Ferraroni et al. (1977) acharam seis casos suspeitos de moléstia de Chagas no alto rio Negro. Em estudos subseqüentes não encontraram o agente Trypanosoma cruzi no sangue humano nem nos vetores (os triatomas) capturados no local (Almeida \& Nunes de Mello, 1978). Outros estudos iguais têm demonstrado triatomíneos infestados com Trypanosoma cruzi na Reserva Florestal Ducke (Almeida \& Machado. 1971).
Ferraroni \& Marzochi (1978) mostraram altos índices de reaçōes sorológicas positivas para toxoplasmose na Amazônia e nos animais domésticos, especialmente gatos. Estudos sorológicos para o agente de sífilis, e de Trichomoniase vaginal en gestantes de Manaus, mostrando taxas de $10 \%$ no primeiro caso e $64 \%$ no segundo (Ferraroni, 1977; Ferraroni et al, 1978).

Em inquérito realizado pelo Departamento Nacionai de Endemias Rurais, os pesquisadores Lacerda e Rachou, demonstraram que Codajás possuia a maior incidência de Mansonella ozzardi no Estado do Amazonas, com um índice de microfilaremia igual a $23,9 \%$. Abordando o mesmo problema, Oliveira (1959b) estudou cinco bairros da cidade de Manaus, examinando 1786 pessoas e encontrou apenas nove portadores de Mansonella ozzardi. Destes casos, provou-se que apenas três eram autóctones, sendo os demais, importados das margens do rio Solimões (Codajás). Diante de tais circunstâncias, as atenções do INPA se voltaram para Codajás com o empenho de estudar o problema. Duas expedições foram então realizadas à referida localidade. Realizaram estudos em $83 \%$ da população, (1.080 pessoas) sendo encontrados 443 casos positivos, constatando o mais alto índice de microfilaremia do Estado $(41,4 \%)$. Batista \& Oliveira, (1960) observaram ainda que os maiores índices de microfilaremia, foram encontrados justamente naquelas localidades cujas casas ficavam mais próximas da mata e os seus residentes eram quase todos lavradores, o que induziu aos autores a admitirem a possibilidade de um transmissor silvestre.

Em 1960, os pesquisadores Batista et al. (1960a) preocuparam-se em estudar a patogenicidade da Mansonella ozzardi e a sintomatologia da monsonellose. Chegaram a conclusão de que o referido agente, determina uma moléstia benigna, de evolução lenta, pouco sintomática e de prognóstico favorável. Os principais sintomas observados foram; dores artículares, frieza nas pernas, placas eritemato-pruriginosas, adenite ingno-crural e dor de cabeça. Sendo que a referida sintomatologia é mais exuberante naqueles pacientes portadores de mais alta microfilaremia. Posteriormente, Oli- 
veira, (1961b) admitia a hipótese de ser a sintomalogia da mansonellose na maioria dos casos a tradução de uma reação provocada pelas reinoculações repetidas de formas infestantes de $M$. ozzardi em organismos previamente sensibilizados.

Todos os pesquisadores do INPA que têm estudado $\sigma$ assunto, foram unânimes em apontar uma alta incidência de parasitoses intestinais, na população amazônica, tanto em Manaus, como em diferentes localidades do interior (Moraes, 1959; Montenegro et al. 1963). Oliveira (1959a) afirmava ser a verminose, entre escolares de Manaus, um seríssimo problema de saúde pública vinte anos atrás, situação esta não muito divergente da realidade atual (Pinheiro et al., 1976).

As diarréias são as maiores causas da mortalidade infantil na região (Giugliano et al., 1978). Giugliano et al. (1977) estudando a etiologia das diarréias, encontrou um terço de uma população de lactentes, de um bairro pobre de Manaus, com diarréia. Foram identificados os prováveis agentes bacterianos em $32 \%$ dos estudados. Neste estudo, foram identificadas as primeiras cepas de Escherichia coli enterotoxigênica na região Amazônica (Giugliano et al. 1978).

Machado (1971) apontava a prevalência da hanseniase no Amazonas, como uma das mais altas do Brasil (6,4/1000 hab.) sendo superada, apenas pela registrada no Acre, onde a incidência variava entre 6,1 e 102,9/ 100.000 hab.. Especificamente em Manaus, ele relatou a existência de 2.331 hansenianos, com uma prevalência de 9,6/100 hab..

Machado, referia-se ao Amazonas, como um persistente desafio aos especialistas em controle de Hanseníase e interpretava a elevação da prevalência, como resultante de: (a) migração de doentes; (b) contaminação de imigrantes sadios, pelos doentes existentes no local; (c) promiscuidade e baixo padrão sanitário nas áreas pioneiras e (d) ausência de um plano efetivo de controle. Decorrida quase uma década, o problema persiste ainda (Salém, 1981).

Os trabalhos iniciais da Micologia Médica do INPA, foram caracterizados pelos registros e estudos de casos clínicos de micoses, nota-

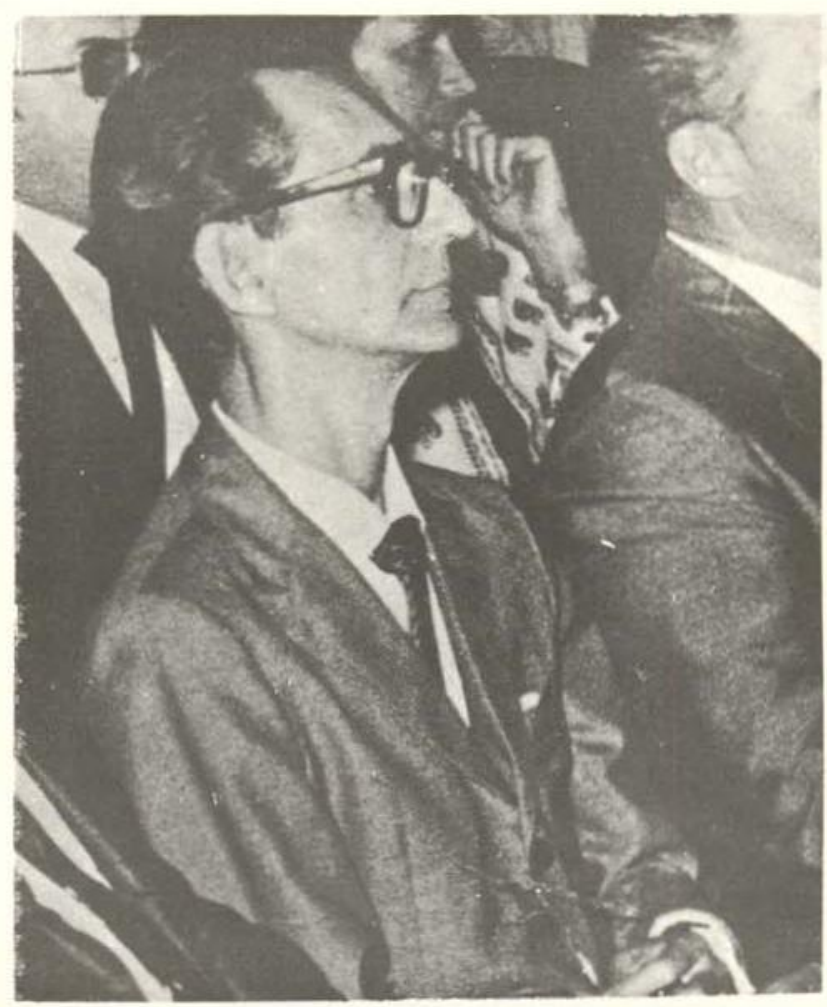

Fig. 1 - Djalma Batista. Eminente pesquisador dos problemas de saúde do homem na Amazônia. Exemplo a ser seguido pela nova geraçăo de pesquisadores do INPA.

damente em Blastomicoses queloideana, que foram sumarizados numa apresentação em 1967, por Moraes e Ferreira, sobre a situação global das endemias micóticas na Amazônia. Nesse trabalho apresentado ao Simpósio sobre a Biota Amazônica, (Moraes \& Ferreira, 1967) assinalaram a presença das seguintes micoses profundas em ordem decrescente de prevalência: Esporotricose, Cromomicose, Doença de Jorge Lobo, Blastomicose Sul-Americana, Rinosporidiose, Micetoma e Histoplasmose. Das micoses superficiais, registraram a Pityriasis versicolor $\mathrm{e}$ as dermatofitoses causadas por Trichophyton tonsurans e T. mentagrophytes, como as mais comuns e a piedra preta, tinea nigra e tricomicose nodular as menos prevalentes.

Posteriormente, já na década de 70 , iniciaram-se os levantamentos epidemiológicos das micoses, com a finalidade de determinar a exata endemicidade dessas infecções, aliando-se a estudos sistemáticos sobre a ecologia dos fungos patogênicos. Foram então levados a 
efeito em Manaus e em cidades do interior do Estado, inquéritos micológicos, sorológicos e imunológicos sobre as dermatofitoses: Pityriasis versicolor, esporotricose, histoplosmose, paracoccidioidomicose e aspergilose. Confirmaram-se o $T$. tonsurans como o patogeno predominante nas dermatofitoses humanas e o único agente causador de "Tinea capitis "nas crianças e o $T$. mentagrophytes o mais encontrado dermatófito nos adultos. Verificou-se também uma prevalência de $15,2 \%$ para a Pityriasis versicolor, a dermatomicose mais comum na região (Fonseca et al., 1975) .

Apesar da diferença no número de casos clínicos, a esporotricose não é diferente da histoplasmose na magnitude da endemicidade. Os índices de positividade à reações intradérmicas à esporotriuina e histoplasmina varianı de $40,8 \%$ a $50,1 \%$ nas populações normais (Fonseca et al., 1973; Mok \& Fava Netto, 1978). $A$ baixa reatividade intradérmica à paracocci-

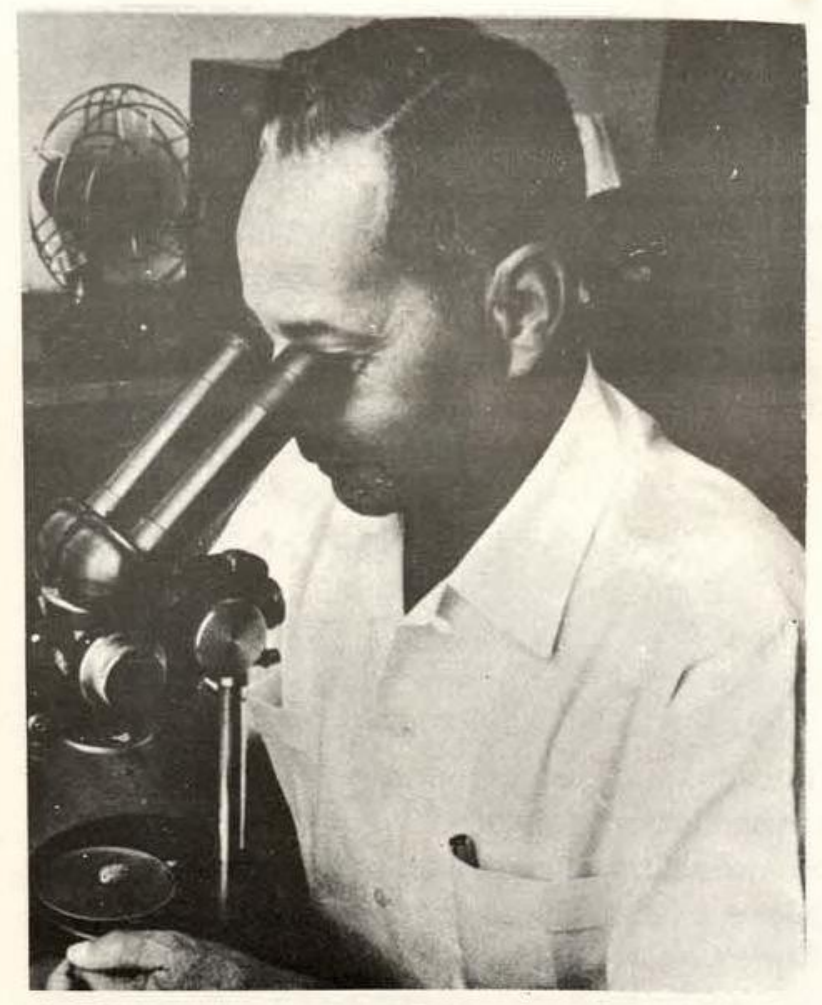

Fig. 2 - Nelson Cerqueira. Um dos expoentes da Entomologia médica da Amazônia. Laureado com o prêmio "Carlos Chagas" da Academia Nacional de Medicina, em 1959, pela determinação da transmissão da Mansonella ozzardi. diơidina $(13,9 \%)$ na população assintomática (Mok \& Fava Netto, 1978) é acompanhada por um pequeno índice sorológico de 1,8\% em pacientes com doenças pulmonares (Mok et al., 1979). A escascez de micoses pulmonares na região Amazônica foi evidenciada por baixíssimos indices de reação sorológica: $1,4 \%$ de aspergilose, $0,4 \%$ de histoplasmose e $0,2 \%$ de esporotricose em pacientes com infecções pulmonares (lbid.) .

As pesquisas sobre a ecologia dos agentes de micoses visaram identificar os focos naturais extra humanos de fungos patogênicos e determinar o papel dos reservatórios na transmissão das micoses. O solo, foi incriminado como reservatório de $T$. mentagrophytes, $\mathrm{Mi}$ crosporum gypseum (Vilela \& Moraes, 1962; Fonseca, 1976) e M. amazonicum (Moraes et al., 1967; Moraes, 1975; Castrillón et al., 1976). A presença de Histoplasma capsulatum no solo é uma questão ainda em aberto, visto que o fungo com características similares à descrição do patógeno, não apresentou o caráter dimorfotípico desse aumiceto (Moraes \& Almeida, 1979). Os pelos de animais silvestres, também foram identificados como habitat do dermatófito zoofilico M. cookei (Moraes \& Almeida, 1978). Num recente e exaustivo estudo, o solo e os morcegos amazônicos foram detectados como fontes naturais de muitos fungos leveduriformes de importância médica, incluindo Wangiella dematitidis, agente de faeohifomicose isolado pela primeira vez da natureza por Reis \& Mok, (1979). Com estudos posteriores de Mok (1981), os estudos biológicos de $W$. dermatitidis demonstraram interações entre o potencial patogênico e seu hospedeiro, sua presença no ambiente $e$ as atividades humanas (Mok \& Luizão, 1980; Mok, 1981) .

A Tinea nigra palmaris, foi registrada apenas uma vez no Amazonas (Fonseca \& Pecher, 1971). O seu agente Exophiala (Pullularia) werneckii foi isolado de peixe salgado, registro esse que evidencia a natureza halofílica desse fungo (Mok, et al., 1981). A blastomicose queloidena por ser uma micose amazônica, tem sido objeto de interesse de pesquisadores do INPA. Além do registro de novos casos com descrição das formas clínicas, anatopato- 
logia, epidemiologia a micopatologia (Moraes \& Oliveira, 1962; Machado, 1972a e 1972b). Num exaustivo estudo sobre o agente etiológico (Fonseca \& Lacaz, 1971) permitiu a redescrição do Paracoccidioides loboi com base nos aspectos morfológicos anatomapatológicos de fungos. A Paracoccidioidomicose foi registrada em Manaus por Moraes \& Ferreira (1967) e Castrillón et al. (1972) e a resistência "in vitro" do Paracoccidioides brasiliensis à Anfotercina B, testada por Castrillón \& Minami (1971).

\section{ESTUDOS RELACIONADOS AOS VETORES DOS PARASITAS}

Dentre os vetores dos parasitas de interesse médico os Artrópodes, especialmente os insetos hematófagos, representam o grupo de maior importância. Considerando que os insetos compõem a maior parte da biomassa da Amazônia, o envolvimento de estudos entomológicos dentro do Departamento Médico, do INPA, sempre tem sido um componente forte. Apenas dois grandes grupos de insetos hematófagos criaram interesse para estudos no sentido médico. Os dipteros (piuns, mutucas e mosquitos) e os hemipteros (barbeiros, piolho de piaçaba). No relato a seguir, as doenças e/ou parasitas que são citados não são necessariamente os únicos transmissiveis por estes insetos, mas sim, os que deram origem aos estudos. Para ajudar o leitor, talvez seja propício elucidar o esquema de atividades associadas à entomologia médica. Após se estabelecer que um inseto é vetor de um determinado parasita, poderá ser descrito a espécie em suas várias formas morfológicas, caso não exista o mesmo. Posteriormente poderá ser feita a distribuição geográfica da espécie aclopada ou não aos estudos de niveis de infecção e/ou agente. Estudos biológicos dos insetos, para descobrir seu ciclo de vida são necessários para a formulação de hipóteses de intervenção no ciclo, seja de controle biológico, químico ou mecânico.

Os trabalhos de Nelson Cerqueira mere. cem referência especial, pois representam um esforço admirável para o conhecimento da Ta.

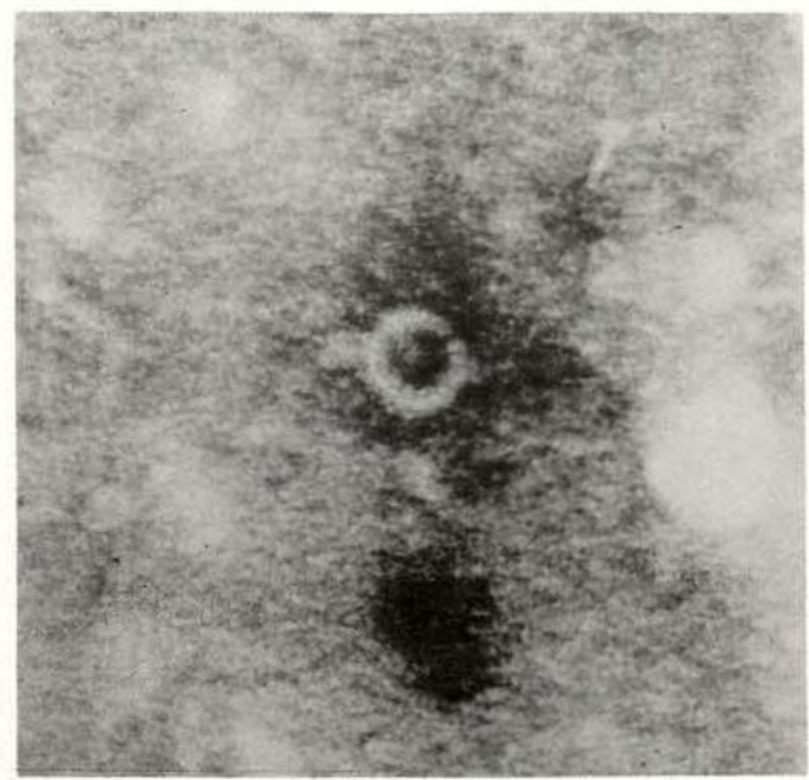

Fig. 3 - Rotavírus - Aumentado $175.000 \mathrm{X}$. Agente etiológico de Diarreia. Uma das maiores causas de morbilidade e mortalidade infantil na Amazônia. Atualmente em estudo na Divisão de Microbiologia, Alimentaçăo e Nutriçāo.

xionomia, Ecologia e Biologia dos mosquitos da Amazônia. O trabalho que o notabilizou foi justamente na pesquisa do vetor da Mansonella ozzardi (Cerqueira, 1959). O autor pesquisou hematófagos silvestres em Codajás e encontrou naturalmente infestados exemplares de simulídeos $(18,5 \%)$ e tabanídeos $(21,4 \%)$. Prosseguindo nos estudos fazendo capturas com iscas humanas, conseguiu o citado pesquisador, estabelecer o ciclo completo da evolução do parasita, no organismo do Simulium amazonicum. Sem dúvida alguma, foi Cerqueira, um expoente da Taxionomia em sua época. A ele, devemos a primeira láurea conquistada pelo INPA, com o prêmio "CARLOS CHAGAS" - da Academia Nacional de Medicina em 1959. pela determinação da transmissão da Mansonella ozzardi, na Amazônia brasileira, através do Simulium, vulgarmente conhecido por "pium".

Apenas na família culicidae os nossos conhecimentos têm chegado ao nível de Biologia de Insetos. Os trabalhos de Cerqueira (1961) deram condições para que Charlwood, após um hiato de tempo, desse prosseguimento na identificação e Biologia dos Culicidae (Charlwood, 1979; Wilkes \& Charlwood, 1979; Rafael \& 


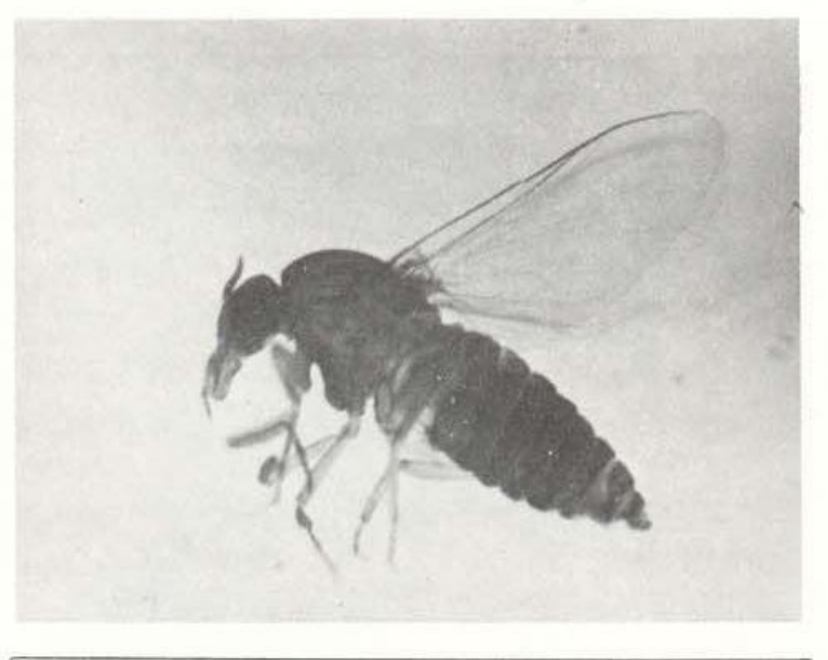

Fig. 4 - Inseto (Diptera). Transmissor de Filária (Mansonella ozzardi), pertencente à familia Simulidae, uma das quais Nelson Cerqueira orientou seus estudos.

Charlwood, 1980; Charlwood \& Wilkes, 1979; Hayes \& Charlwood, 1977; Charlwood \& Hayes, 1978; Hayes \& Charlwood, 1979).

Um acontecimento lamentável foi o que aconteceu com Mohamed Rabbani, que iniciou estudos importantes da Citogenética dos Anopheles (Kreutzer et al., 1976) vetor da malária, da qual ele faleceu. Seus estudos foram continuados por Santos (Tadei et al., 1980, Narang et al., 1979; Santos, 1980).

$\mathrm{Na}$ família Psychodidae, onde se encontram espécies capazes de transmitir vários parasitas causadores das Leishmaniose, os estudos na maioria se restringem à descrição das espécies e suas infestações e comportamento (Almeida, 1970a e 1970b; Young \& Arias, 1977; Arias \& Freitas, 1977a; Arias \& Freitas, 1977b; Biancardi, 1981) .

Os trabalhos relacionados aos Simulídeos iniciados por Cerqueira ainda estão no estágio de estudos descritivos e zoogeográficos (Gorayeb \& Pinger, 1978), com um único estudo biológico visando possíveis métodos de controle biológico, desde os potenciais vetores da Mansonella ozzardi e Onchocerca volvulus (Mello, et al., 1973; Mello \& Silva, 1974; PyDaniel, 1979; Lacey \& Charlwood, 1980; Py-Daniel \& Shelley. 1980). Este mosquito simplesmente pela sua insistência a nível de picada representa um dos maiores incômodos ao caboclo do interior.

\section{ESTUDOS RELACIONADOS AO HOMEM}

Montenegro estudou a composição étnica das populações da Amazônia, através do índice siclêmico (Montenegro, 1959), e dos grupos sangüineos (Montenegro, 1960a; Junqueira et al., 1962). Mostraram a forte influência do branco em Manaus e do negro em Codajás, ligados a forte influência indígena, em ambos os lugares. Montenegro (1959) assinalou também as baixas taxas de hemoglobina em caboclos da região. Posteriormente o referido autor, estendeu o mesmo estudo à pessoas de alto nível sócio-econômico de Manaus, para confirmar a possibilidade de taxas de hemoglobina aceitas como normais internacionalmente, também neste clima tropico-húmido (Montenegro, 1961). Enfatizou ainda, que o status sócioeconômico, era um fator importante associado à anemia (Montenegro, 1960b) e que a suplementação de ferro, não resolve o problema. sem que antes, sejam tratados os parasitas intestinais (Montenegro apud Batista, 1964).

Samuel Aguiar, investigando o metabolismo basal em pessoas de classe sócio-econômica alta de Manaus encontrou índices aceitos como normais internacionalmente (Aguiar, 1966). O mesmo autor verificou também, que as estaturas em crianças de Codajás eram inferiores daquelas registradas nas crianças do Baixo Amazonas e Sul do país. Contente (1968) numa pequena amostra de crianças de bairros pobres de Manaus, encontrou baixa estatura, baixos níveis de hemoglobina e sinais clínicos sugestivos de deficiência de riboflavina. Dados semelhantes foram reportados por Giugliano, e colaboradores (Giugliano et al., 1977), Giugliano \& Shrimpton, 1978) que também verificaram uma alta prevalência de sinais clínicos sugestivos de hipovitaminose A. A freqüência de baixo peso ao nascer em crianças de Manaus, é $2 / 3$ dos valores encontrados em crianças do Nordeste, (Giugliano et al.. 1979).

Os estudos de padrão alimentar em lactentes mostraram que a amamentação estava em declínio na cidade de Manaus, em comparação com o interior (Shrimpton \& Giugliano, 1977; Giugliano et al., 1978). As misturas alimentares infantis, usadas para suplementar e subs- 


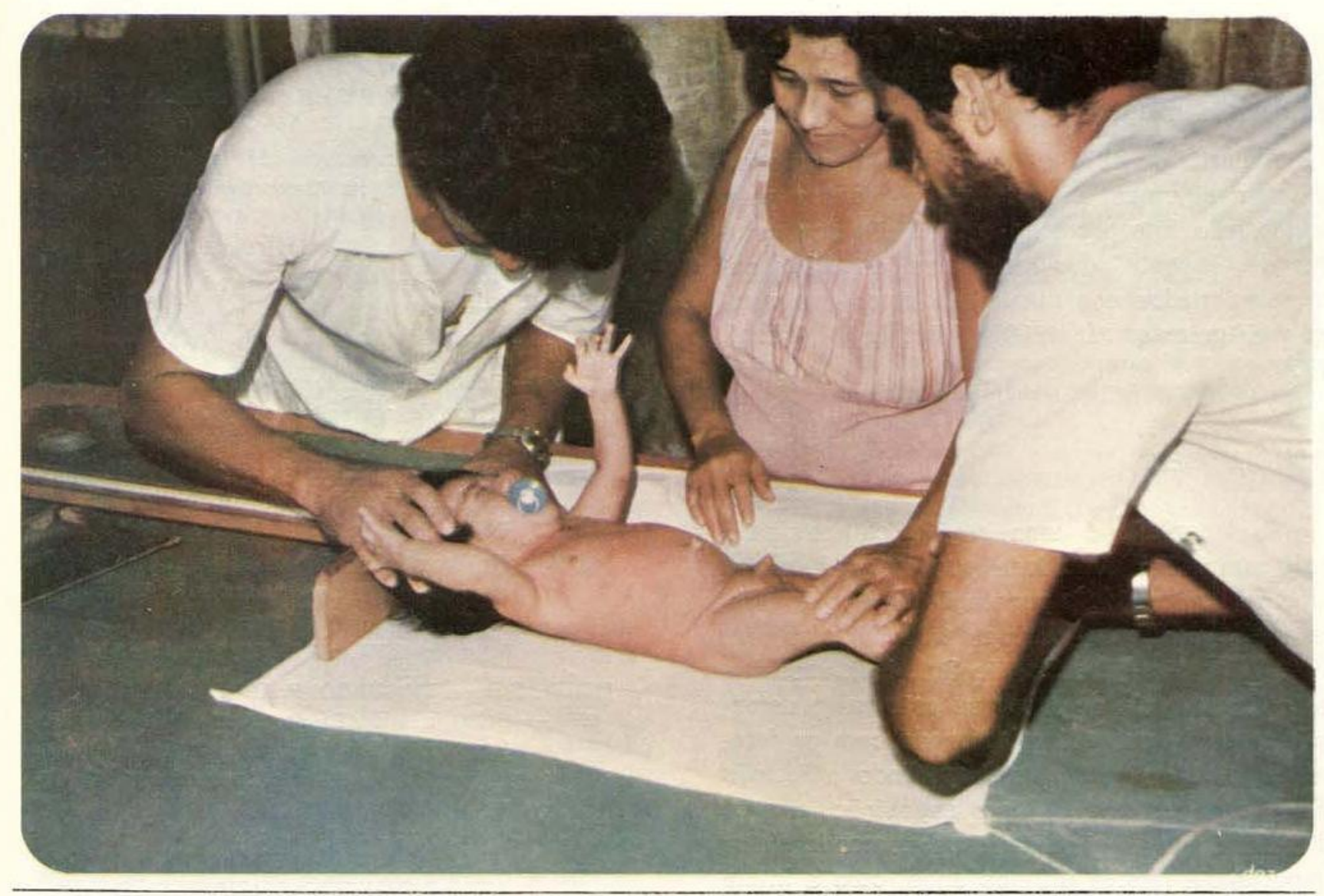

Fig. 5 - Estudo longitudinal, de crianças do bairro do Coroado - Manaus, durante o primeiro ano de vida. Visa o conhecimento da nutriçāo, desenvolvimento e infecçōes acometidas em seu habitat.

tituir o leite materno, foram consideradas mais deficientes em zinco, do que em proteinas. Sendo que a razão zinco-energético, mostrouse suficientemente baixa, para interferir no crescimento normal do lactente (Shrimpton, 1980)

Lira (1960) na expedição da $2^{\text {a }}$ Divisão à Codajás, em 1958, foi o primeiro pesquisador do Departamento a preocupar-se com o padrão alimentar regional. Ele constatou que a dieta em Codajás baseava-se na farinha de mandioca, banana, pão, peixe e banha, e o consumo de carne era insignificante. Uma análise dos dados sobre o consumo de alimentos em familias de Manaus, mostrou que sua dieta difere do restante do Brasil, pelo baixo consumo de verduras e frutas (Shrimpton \& Giugliano, 1979). Dos dez nutrientes considerados na dieta de Manaus, o zinco e vitamina $A$ foram os mais deficientes, enquanto o ferro era adequado, apesar do alto índice de anemia local (Shrimpton, 1980).
Shrimpton (1980) investigou os níveis séricos de zinco em 335 operários de baixa renda em Manaus e mostrou que eram insignificantemente relacionados à renda e ao consumo de zinco nas últimas 24 horas. Trinta e quatro por cento dos operários de famílias de baixa renda, $26 \%$ das de média renda e $17 \%$ dos de alta renda tinham níveis de zinco sérico inferiores aos limites de normalidade assumidos (75 ug/ $100 \mathrm{ml}$ ). Os níveis de zinco no cabelo mostraram uma correlação significativa com níveis sáricos de zinco em operários näo parasitados (a nivel intestinal). Os niveis séricos de vitamina A nos mesmos operários foram analizados, demonstrando que $3 \%$ destes tinham niveis séricos deficientes e $15 \%$ abaixo dos niveis considerados aceitáveis (Marinho et al:, 1981). Salem (1981) não achou correlação entre os niveis de blastorgenese com os níveis séricos de vitamina $A$ e de retinol em hansenianos virgens de tratamento. Surpreendentemente no grupo de controle houve uma corre- 
lação inversamente significativa, entre o nível de zinco sérico e a blastogenese, fato que precisa de maiores estudos.

A partir de 1977 o Departamento começou a fazer algumas análises de alimentos para melhorar a interpretação e precisão dos estudos de "ingesta" dos nutrientes, especialmente relacionados aos nutrientes zinco e vitamina A (Aguiar et al., 1980). Marinho \& Arkcoll 1981.

\section{CONCLUSÕES}

A Amazônia é uma região caracterizada pela sua baixa densidade populacional, muito embora, tenha sido a população da Região Norte triplicada nestas últimas três décadas, em virtude do êxodo da Região Sul. Daí, ressaltase a importância de saber se este vazio demográfico do Norte está relacionado com os fatores ambientais regionais, impedindo a fixação do homem na região. Esta suposição, a cada dia passa a ser mais enfatizada; tomando maior importância nestes últimos anos, com a intenção do Governo Federal em colonizar esta imensa região. Os possiveis fatores ambientais determinantes de saúde, nesta região tropical-úmida, incluem a água, o ar, os perigos biológicos, a estrutura e composição do solo, luz solar, vegetação, alimentação, e demais fatores. Dependendo do equilíbrio ou desequilíbrio do ser vivo, frente a esses fatores, além daqueles criados pelo homem, haverá saúde ou enfermidade.

Acreditamos que esta revisão bibliográfica mostre que a contribuição do Conselho Nacional de Desenvolvimento Científico e Tecnológico (CNPq) através do Instituto Nacional de Pesquisas da Amazônia (INPA) ao desenvolvimento da ciência na área de Medicina Tropical e Saúde da Amazônia é algo de orgulhar-se.

Djalma Batista (1964) afirmou que a Hinterlândia Amazônica poderá ser dominada pela técnica e pela ciência, e o homem pode e deve aprimorar a sua cultura pela educação e pela higiene, dentro de uma sociedade regida por novas diretrizes econômicas. Esperamos ter condições para que possamos tornar esta afirmação uma realidade.

\section{REFERENCIAS BIBLIOGRAFICAS}

AGUIAR, J.P.L.; MARINHO, H.A. REBELLO, Y.S.; SHRIMPTON, R.

1980 - Aspectos nutritivos de alguns frutos da Amazônia. Acta Amazonica, 10 (4): 755-758.

AGUIAR, Samuel.

1966 - 119 basal metabolism experiments in Manaus-Amazonas. O Hospital, 70 (5): 1319 . 1325.

ALMEIDA, F.B.

1970a - Flebótomos da Amazônia II. Sobre a ocorrência de anomalias na genitalia masculina em Lutzomya anduzei (Rozeboom 1942) e Lutzomyia rorotaensis (Floch e Abonnenc, 1944). (Diptera, Psychodidae). Boletim do INPA; Série Patologia Tropical, 1: 1-4.

1970b- Flebótomos da Amazônia II. Sobre a presença de Lutzomyia anduzei (Rozeboom. 1942). No Brasil (Diptera Psychodidae). Boletim do INPA; Série Patologia, 3: 1-16.

ALMEIDA, F.B. \& MACHADO, P.A.

1971 - Sobre a infecção do Panstrongylus geniculatus pelo Trypanosoma cruzi em Manaus Amazonas Brasil. Acta Amazonica, 1 (2): 71 75.

ALMEIDA, F.B. \& NUNES DE MELLO, J.A.

1978 - Sobre a ocorrência de moléstia de chagas no Estado do Amazonas, Brasil. Acta Ama. zonica, 8 (3): 595-599.

ARIAS, J.R. \& FREITAS, R.A.

1977a - Flebótomos da Amazônia Central do Brasil. 1. Resultados obtidos das capturas feitas com isca humana (Diptera, Psychodidae) (Patologia). Acta Amazonica, 7 (4): 507-527.

$1977 \mathrm{~b}$ - On the vectores of cutaneous Leishmaniasis in the central Amazon of Brazil. I. Preliminary fingings. Acta Amazonica, 7 (3): 293294.

ARIAS, Jorge R.; MENESES, H.; FREITAS, Rui A. de

1976 - Evaluation of the "PE' vaccine against two strains of Leishmania sp. in hamsters. Acta Amazonica, 6 (4): 483-485.

BATISTA, D.

1964 - Da habilidade da Amazônia. Rio de Janeiro, Serv. Graf. do IBGE, 39 p. (Cadernos da Amazônia).

1976 - O Complexo da Amazônia; Análise do processo de desenvolvimento. Rio de Janeiro. Conquista, 1976. 292 p.

BATISTA, D. \& OLIVEIRA, W.R.

1960 - Epidemiologia da monsonelose em localidade do interior do Amazonas. Rev. Ass. Médica Bras. São Paulo, 6 (3): 176-184.

BATISTA, D.; OLIVEIRA, W.R.; RABELLO, V.D.

1960a-Codajás: Comunidade Amazônica. Revista Bras. de Geografia, 22 (3) 321-342. 
1960b - Estudo da patogenicidade da Mansonella ozzardi e da sistomalogia de mansonelose. Rev. do Inst. de Med. Tropical de São Paulo, 2 (5): 281-289.

BIANCARDI, Ceclie Ma. A. Barbosa

1981 - Aspectos da epidemiologia da Leishmania Cutanea na Rodovia BR-364, Território Federal de Rondônia. Tese de Mestrado PósGraduação INPA-FUA, Manaus.

CASTRILLÓN, A.L.; CARVALHO, R.F.; BORBOREMA,

C.A.; PECHER, S.A.

1972 - Paracoccidioidomicosa na Amazônia; registro de um caso. Acta Amazonica, 2 (3): 55-58.

CASTRILLÓN, A.L. \& MINAMI, P.S.

1971 - Resistência do Paracoccidioides brasiliensis "in vitro" a anfotericina B. Rev. Microbiologia, 2 (2): 93-96.

CASTRILLON, A.L.; MORAES, M.A.P.; FURTADO,

M.S.S.

1976 - Isolamento de Cicrosporum amazonicum do solo do Estado do Amazonas, Brasil. Acta Amazonica, 6 (3): 487-490.

CERQUEIRA, N.L.

1959 - Sobre a transmissão da mansonella ozzardi (I e II Notas). Jor. Bras. de Medicina, 1 (7): 885-914.

1961 - Distribuição geográfica dos mosquitos da Amazônia. Rev. Bras. de Entomologia, 10: 111-168.

CHARLWOOD, J.D.

1979 - Estudos sobre a biologia e hábitos alimentares de Culex quinquefasciatus Say de Manaus, Amazonas, Brasil. Acta Amazonica, 9 (2): $271-278$

CHARLWOOD, J.D. \& HAYES, J.

1978 - Variações geográficas no ciclo de picada do Anopheles darlingi Root no Brasil. Acta Amazonica, 8 (4): 601-603.

CHARLWOOD, J.D. \& WILKES, T.J.

1979 - Studies on the age compositon of Anopheles darlingi Root in Brazil. Bulletin of Entomological Ressearch, 69 (2): 232-233.

CONTENTE, J.J. Sousa

1968 - Estudo clínico-nutricional em menores da cidade de Manaus. Rev. Assoc. Med Bras., 1: 203-219.

DEANE, L.M.; FERREIRA NETO, J.; CERQUEIRA, N.L. 1966 - Observações preliminares sobre malária de macacos no Estado do Amazonas. Rev. Bras. de Biologia, 26 (4): 405-412.

FERRARONI, J.J.

1977 - A possivel incidência das sífilis em gestantes da cidade de Manaus. Acta Amazonica, 7 (2): 255-262.

FERRARONI, J.J.; CARNEIRO, O.S.; PRIMO, L.C.

1978 - Freqüência da trichomoniose vaginal em gestantes atendidas na maternidade Escolar Ana Nery, Manaus Amazonas. Acta Amazonica, 8 (2): 217-224.
FERRARONI, J.J. \& DOURADO, H.V.

1977 - Uso da minociclina endovenosa no tratamento da malária por plasmodium falciparum. Acta Amazonica, 7 (2): 263-272.

FERRARONI, J.J.; MARZOCHI, M.A.

1978 - Taxoplasmose em animais domésticos e silvestres de Manaus-Amazonas. Acta Amazonica, 8 (1): 83-89.

FERRARONI, J.J.; NUNES DE MELLO, J.A.;

CAMARGO, M.E.

1977 - Moléstia de chagas na Amazônia. Ocorrência de seis casos suspeitos, autoctones, sorologicamente positiva. Acta Amazonica, 7 (3): $438-440$

FONSECA, O.J.M.

1976 - Fungos aratinofílicos do solo de Manaus. Acta Amazonica, 6 (1): 63-65.

FONSECA, O.J.M.; CASTRILLÓN, A.L.; FERRARONI, J.J. 1975 - A "Pityria Versicolar" no Estado do Amazonas. Acta Amazonica, 5 (2): 195-198.

FONSECA, O.J.M. \& LACAZ, C.S

1971 - Estudo de sultura isoladas de blastomicose quilodiforme (doença de Jorge Lobo) denominação do seu agente etiológico. Rev. Inst. Med. Trop. São Paulo, 13: 225-251.

FONSECA, O.J.; LACAZ, C.S.; MACHADO, P.A.

1973 - Inquéritos imuno-alérgico na Amazônia; resultados preliminares. Rev. Inst. Med Trop. S. Paulo, 15: 406-416.

FONSECA, O.J.M. \& PECHER, S.A.

1971 - Tinea nigra no Amazonas. Acta Amazonica, 1 (3): $55-57$.

GIUGLIANO, L.G.; GIUGLIANO, R.; PINHEIRO, M.F.S.

1977 - Bactérias enteropatogênicas em lactentes de um bairro de Manaus. Acta Amazonica, 7 (3): 395-400.

GIUGLIANO, L.G.; NAKAGIMA, G.S.; GIUGLIANO, R.; SHRIMPTON, R.

1978 - E. Coli interotoxigênica isolada em lactentes em Manaus, Amazonas, Brasil. Rev. Microbiologia, 9: 198-201.

GIUGLIANO, R.; ALBUQUERQUE, Helyde C.R.;

SHRIMPTON, R.

1978 - Estudo antropométrico, clínico e de padrōes alimentares em escolares de Manaus, 1976. Acta Amazonica, 8 (1) 75-82.

GIUGLIANO, R. \& SHRIMPTON, R.

1977 - Estudos antropomético e clínico do Estado nutricional em um grupo de pré-escolares de Manaus. Acta Amazonica, 7 (3): 389-394.

GIUGLIANO, R.; SHRIMPTON, R.; ARKCOLL, D.B.;

GIUGLIANO, L.; PETRERE, M

1978 - Diagnóstico da realidade alimentar e nutricional do Estado do Amazonas, 1978. Acta Amazonica, 8 (2) Supl. 254 p.

GIUGLIANO, R.; SOUZA, J. Viana de; SHRIMPTON, R.; GIUGLIANO, L.G.

1979 - Levantamento da freqüência do baixo peso de nascimento em Manaus, 1976. Acta Amazonica, 9 (1): 109-116. 
GORAYEB, I.S. \& PINGER, R.R.

1978 - Detecção de predadores naturais das larvas de Simulium fluvinotum Cerq. e Mello, 1968 (Diptera: Nematocera). Acta Amazônica, 3 (4): 629-637.

HAYES, J. \& CHARLWOOD, J.D.

1977 - O Anopheles darlingi evita o DDT numa área de malária resistente a drogas. Acta Amazonica, 7 (2): 289.

1979 - Dinâmica estacional de uma população de Anopheles darlingi numa área andêmica de malária no Amazonas. Acta Amazonica, 9 (1): $79-86$.

JUNQUEIRA, P.C.

1962 - Grupo sanguíneo de nordestinos. Anais da Academia Bras. de Ciências, 54 (1): 143152.

KREUTZER, D. KITZMILLER, B.E. \& RABANI, M.G.

1976 - Cytogenetically distinguishable sympatric ans allopatric populations of the mosquito Anopheles albitarsis. Acta Amazonica, 6 (4): 473-481.

LACEY, L.A. \& CHARLWOOD, J.D.

1980 - On the biting activities of some onthropophilic Amazonian Simulidae (Diptera). Bull. Ent. Res. 70, 495-509.

LIRA, M.B.

1960 - Levantamento de dados alimentares em cidades do interior Amazônico. Revista Bras. de Medicina, 17 (7): 637-638.

MACHADO, P.A.

1971 - Relator oficial "Hansianiase na grande regiāo norte". Boletim do Serv. Nac. de Lepra, $30(1 / 2): 9-36$.

1972a - Polimorfismo das lesões dermatológicas na blastomicose de Jorge Lobo entre índios Caiabi. Acta Amazonica, 2 (2): 93-98.

$1972 b$ - Regressão espontânea de lesões musculosas na blastomicose Jorge Lobo. Acta Ama. zonica, 2 (1): 47-50.

MARINHO, H.A.; FRANÇA, T.S.; REBELLO, Y.S.;

SHRIMPTON, R.

1981 - Niveis séricos de vitamina " $A$ ' em operários de Manaus. Acta Amazonica (no prelo).

MARINHO, H.A. \& ARKCOLL, D.B.

1981 - Estudos sobre o caroeteno em algumas variedades amazônicas de mandioca. (Ma. nihot esculenta crants). Acta Amazonica (no prelo).

MELLO, J.A.N.; ALMEIDA, F.B.; DELLOME, J.

1973 - Simulidae do Amazonas VI. Descrição do Simulium metuoco sp. N. (Diptera: Nematocera). Acta Amazonica, 3 (3): 59-62.

MELLO, J.A.N. \& ALMEIDA, F.B.

1974 - Simulidae da Amazônia. VII. Acta Amazo. nica, 4 (1) 65-67.

MELLO, J.A. \& SILVA, E.V.

1974 - Simulidae da Amazônia. VIII. Acta Amazonica (2): 69-71.
MOK, W.Y.

1981 - Ecology of wangiella dermatitidis in the Amazon Basin. Proc. V PAHO Symp. on Mycoses, (no prelo).

MOK, W.Y.; AYRES, H.L.; MCMILLEM, S.

1979 - Levantamento sorológico de quatro micoses profundas no Estado do Amazonas, Brasil. Acta Amazonica, 9 (1): 75-78.

MOK, W.Y.; CASTELO, F.P.; BARRETO DA SILVA, M.S.

1981 - Ocurrence of Exophiala vernechii on salted freshwater fish osteoglossum. Jour. Food. Tech. (no prelo).

MOK, W.Y. \& FAVA NETO, E.

1978 - Paracoccidioidin and Histoplasmin ski sensitivity in Coari (State of Amazon) Brazil. Am. J. Trop. Med. Hyg., 27: 208-814.

MOK, W.Y. \& LUIZÃO, R.C.C.

1980 - Serologia analysis and pathogenic potencial of wangiella dermatitidis isolated from bats. Micopathologia, 71: $(37 / 38)$,

MONTENEGRO, Luiz

1959 - Indice siclemico com uma comunidade do interior do Amazonas. O Hospital, 55 (2): 273-278.

1960a - Freqüência dos grupos sanguíneos do sistema $A B O$ e do fator $D(R H O)$ em Manaus. Sangre, 5: 191-196.

$1960 \mathrm{~b}$ - Quadro vermelho de uma comunidade do interior do Amazonas. 0 Hospital, 56 (3): 466-506.

1961 - Considerações sobre as taxas de Hemoglobina e de hemácias na Amazônia. O Hospital, 60 (6): 880-893.

MONTENEGRO, Luiz; BATISTA, Djalma; OLIVEIRA,

Wallace

1963 - Novas observaçōes higiênicas-sociais no núcleo agrícola japonês da Estrada ManausItacoatiara, Estado do Amazonas. O Hospital, 63 (6) 1355-1360.

MORAES, M.A.P.

1959 - Inquérito sobre parasitos intestinais na cidade de Codajás, Estado do Amazonas. Rev. Bras. de Med. 16 (7): 19-25.

1975 - The perfect stat of Microsporum amazonicum. Mycologia, 62: 1109-1113.

MORAES, M.A.P.; ALMEIDA, M.M.R.

1976 - Isolamento de Histoplasma capsulatum do solo de Humboldt, Estado do Mato Grosso, Brasil. Acta Amazonica, 6 (4): Supl. 43-47.

1978 - Isolamento de Cicrosporum cookei do pêlo de animais silvestres sapturados na regiäo Amazônica. Acta Amazônica, 8 (1): 99-101.

MORAES, M.A.P.; BORELLI, D.; FED, M.

1967 - Microsporum amazonicum novas espécies. Med. Cut., 2: 281-286.

MORAES, M.A.P. \& FERREIRA, J.L.S.

1967 - Micoses superficiais e profundas na Amazônia. Ata do Simpósio sobre a Biota Ama. zônica, 6: 189-202. 
MORAES, M.A.P. \& OLIVEIRA, W.R.

1962 - Novos casos de micoses de Jorge Lobo entrados em Manaus, Amazonas, Brasil. Rev. Inst. Trop. São Paulo, 4: 403-406.

NARANG, S.; SANTOS, J.M.M.; GARCIA, J.C.;

CRISTKOU, H.D.; NARANG, N.

1979 - Genética de populaçōes de Anofelinos. IV. Estudos eletroforéticos das populaçōes naturais de Anopheles nuneztovari e Anopheles darlingi. Correlação genética entre espécies. Acta Amazonica, 9 (3): 529-542.

OLIVEIRA, W.R.

1959a - Contribuição ao estudo coprologio cidada de Manaus. Brasil Médico, 73 (10/13): 1619.

1959b - Filarioses humanas na cidade de Manaus O Hospital, 52 (2): 301-303.

1961b-Monsonelose e sua sintomatologia. O Hos. pital, 59 (3): 539-543.

PINHEIRO, Ma. F. da S.; VASCONCELOS, J.C.;

WENDEL, D.E.

1976 - Contribuição ao estudo de parasitos intestinais em dois bairros de Manaus-Amazonas. Acta Amazonica, 6 (1): 67-73.

PY-DANIEL, V. \& SHELLEY, A.J.

1980 - Revisão do Simulium spinibranchium Lutz, 1910 (Diptera. Nematocera) com a primeira descrição dos adultos e larvas, e redescrição da pupa. Acta Amazonica, 10 (1): 213-223.

PY-DANIEL, $V$.

1979 - Algumas considerações sobre Simulidae (Diptera, Nematocera). Acta Amazonica, 9 (4): 783-786.

RAFAEL, J.A. \& CHARLWOOD, J.D.

1980 - Idade fisiológica, variação sazonal e periodicidade diurna de quatro populações de tasanidae (Diptera) no Campus Universitário, Manaus-Amazonas-Brasil. Acta Amazonica, 10 (4): 907-927.

REIS, Arthur Cezar Ferreira

1958 - Pesquisas da Amazônia. Objetivos. Funcionamento: Sua contribuição para o conheci- mento realístico da Amazônia, Manaus

Publicação Avulsa, 7) (mimeografado).

REIS, N.R. \& MOK, W.Y.

1979 - Wangiella dematitidis isolated from bats in Manaus, Brasil. Sabourandia, 17: 213-218.

SALEM, J.I.

1981 - Blastogenese em Hanseniase no Estado do Amazonas: "Importância da Vitamina A e do Zinco". Tese de Mestrado INPA/FUA.

SANTOS, J.M.M.

1980 - Aspectos biológicos e isoenzimáticos de Anopheles ( $n$ ) darlingi Root 1926 (Diptera: Culididae). Tese de Mestrado Curso PósGraduação INPA/FUA.

SHRIMPTON, Roger

1980 - Studies on zinc nutrition in the Amazon valley. Tese de Doutoramento. Univ. de Londres. $326 \mathrm{p}$.

SHRIMPTON, R. \& GIUGLIANO, R.

1977 - Nutrição em lactentes de um bairro de Ma* naus-Amazonas. Acta Amazonica, 7 (2): 247 . 253.

1979 - Consumo de alimentos e alguns nutrientes em Manaus-Amazonas, 1973. Acta Amazonica, 9 (1): 117-141.

TADEI, W.P.; SANTOS, J.M.M.; RABBANI, M.G.

1980 - Studies on zinc murition in the Amazon populaçōes naturais de Anopheles darlingi. Ciência e Cultura, 32: 650 .

VILELA, E.M. \& MORAES, M.A.P.

1962 - Isolamento de microsporum gypseum e Trichophyton mentagrophytes do solo de Manaus-Amazonas, Brasil. Rev. Inst. Med. Trop. Sāo Paulo, 4: 299-301.

WILKES, T.J. \& CHARLWOOD, J.D.

¿979 - Um rápido ciclo gonotrófico em Chagasia bonneae Root (Diptera: colicidae) do Brasil. Acta Amazonica, 9 (3): 615-616.

YOUNG, David G. \& ARIAS, J.R.

1977 - Lutzomyia Sand flies in the subgenus Evandromyia Mangabeira with descriptions of a new species from Brazil (Diptera: Psychodidae). Acta Amazonica, 7 (1): 59-70. 